\title{
Self-Sustained Oscillations in a Plasma-Wall System with Strongly Inhomogeneous Diffusion of Charged Particles
}

\author{
M.Z. Tokar \\ Institut für Plasmaphysik, Forschungszentrum Jülich, Association EURATOM-FZJ, Trilateral Euregio Cluster, Germany*
}

(Received 27 July 2005; published 23 December 2005)

\begin{abstract}
A simple system with a hydrogen plasma confined by a magnetic field parallel to the bounding material wall is considered. The charged particles diffuse out of the plasma, recombine on the wall and return into the plasma volume as neutrals, which are ionized by electrons. It is demonstrated that macroscopic selfsustained oscillations are an intrinsic feature of such a system if the diffusion coefficient of charged particles is strongly inhomogeneous in the plasma.
\end{abstract}

DOI: 10.1103/PhysRevLett.95.265002

PACS numbers: 52.35.Py, 52.55.Fa

Introduction. - Plasmas in laboratory devices are complex objects. This complexity manifests itself in different types of self-organization processes and, in particular, by macroscopic self-sustained oscillations seen in the time variation of local and global parameters such as plasma density, temperature, radiation losses, etc. Edge localized modes [1] and divertor oscillations [2] in tokamaks and breathing oscillations in stellarators [3] are examples. Understanding of the nature of these phenomena is crucial for finding more optimal regimes for the operation of modern and future devices and can bring a deeper insight into the nature of complex systems.

The interaction of plasma with the bounding material walls is considered as very essential for the oscillatory behavior described above. In this Letter we analyze a simple system with a hydrogen plasma confined by a magnetic field and enclosed by a wall parallel to the field; see Fig. 1. Electrons and ions diffuse perpendicular to the field out of the plasma volume and recombine on the wall. Owing to diverse processes the generated neutral particles are released back into the plasma; i.e., the so-called particle recycling takes place [4]. In a stationary state the influx of neutrals compensates the loss of ions and electrons from the plasma. The ionization of neutral particles by collisions with electrons provides the source of charged particles in the plasma volume. It will be demonstrated that macroscopic self-sustained oscillations are an intrinsic feature of such a system if the diffusion of charged particles across the magnetic field is sufficiently inhomogeneous in the plasma volume.

Basic equations. - The variation of the densities of neutral and charged particles, $N$ and $n$, respectively, with time $t$ and the coordinate $x$ perpendicular to the magnetic field, is governed by the continuity equations

$$
\begin{gathered}
\frac{\partial N}{\partial t}+\frac{\partial \Phi}{\partial x}=-k_{i} n N, \\
\frac{\partial n}{\partial t}+\frac{\partial \Gamma}{\partial x}=k_{i} n N,
\end{gathered}
$$

where $k_{i}$ is the ionization rate coefficient, and $\Phi$ and $\Gamma$ are the densities of particle fluxes. The influx of neutrals into the plasma is due to desorption of particles captured in the wall material [5]:

$$
\Phi\left(x=x_{w}\right)=-\mu C_{w}
$$

where $\mu$ is the desorption rate coefficient and $C_{w}$ the "surface" density of neutrals in the wall. The time variation of $C_{w}$ is governed by the imbalance between the loss of charged particles out of the plasma to the wall and the rate of neutral desorption:

$$
\frac{d C_{w}}{d t}=\Gamma\left(x=x_{w}\right)-\mu C_{w} .
$$

The flux density of neutrals is determined by taking into account that due to charge exchange with ions the velocity of recycling neutrals changes chaotically many times before they are ionized by electrons [6,7]:

$$
\Phi=-\frac{T}{m\left(k_{i}+k_{c x}\right) n} \frac{\partial N}{\partial x}
$$

with $m$ being the neutral mass and $k_{c x}$ the charge-exchange rate coefficient. We assume the same temperature $T$ for electrons and ions. This is a good approximation, in par-

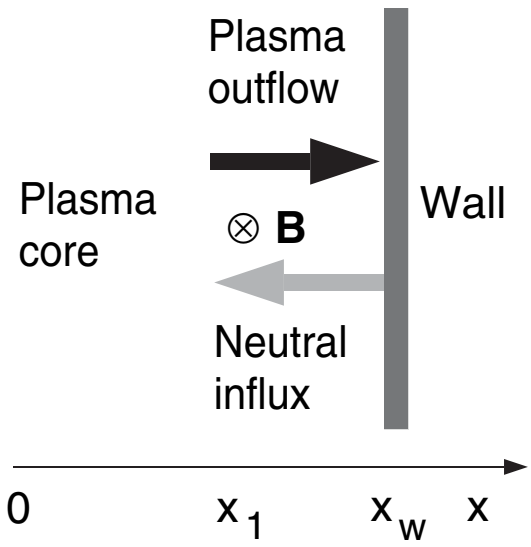

FIG. 1. The near wall region in a magnetized plasma. 
ticular, for tokamak edge plasmas where the temperature equilibration time due to coulomb collisions is much shorter than characteristic times of heating and cooling processes due to, e.g., ionization of recycling neutrals. Under special discharge conditions these temperatures can differ significantly in the plasma core [8].

The flux density of charged particles is governed by perpendicular diffusion:

$$
\Gamma=-D \frac{\partial n}{\partial x} .
$$

The diffusion coefficient $D$ can be spatially very inhomogeneous. In particular, different types of micro-instabilities are responsible for the transport processes in the core and at the edge in tokamak plasmas [9]. Thus, at the very edge, where the plasma temperature is low, the turbulence driven by electron collisions can dramatically increase the particle diffusion [10]. A strong variation of the diffusion coefficient does not contradict a smooth change of such plasma parameters as density and temperature since the plasma micro-instabilities are mostly controlled by the gradients of these parameters [9] Besides, the edge transport is increased significantly by a deliberate magnetic field stochastization [11]. In the following consideration this behavior is described qualitatively by a steplike dependence:

$$
D\left(x \leq x_{1}\right)=D_{0}, \quad D\left(x_{1}<x \leq x_{w}\right)=D_{1}
$$

with constant $D_{0}<D_{1}$; the position $x_{1}$ where the transport changes will be henceforth prescribed but can be determined self-consistently in a more sophisticated consideration.

In the case with a magnetic field parallel to the wall the plasma quasineutrality is broken in the sheath region [12]. In our analysis this region is not taken into account since its width is much smaller than that of the region in question determined by the penetration depth of recycling neutrals.

Stationary states of the plasma-wall system described above are analyzed under simplifying assumptions: (i) the plasma temperature $T$ and, thus, the rate coefficients $k_{i}$ and $k_{c x}$ are independent of $x$, and (ii) the plasma density is zero at the wall because of the fast recombination of electrons and ions there. These restrictions are removed by modelling the time behavior numerically.

For constant $T, k_{i}$ and $k_{c x}$ Eqs. (1) and (5) are combined into the second order equation, $d^{2} N / d u^{2}=N$, with $u=$ $\sigma_{*} \int_{x}^{x_{w}} n d x$ and $\sigma_{*}=\sqrt{m k_{i}\left(k_{i}+k_{c x}\right) / T}$ [7]. This results in an exponential decay of the neutral density and particle fluxes, $N, \Phi, \Gamma \sim \exp (-u)$, and the integral of Eq. (2)into the equation for $u(x): D d^{2} u / d x^{2}=\sigma_{*} \Gamma\left(x=x_{w}\right) \times$ $\exp (-u)$. The latter can be integrated analytically in each $x$ region of constant $D$. A relation between the density of the charged particle outflow to the wall and the particle density $n_{c}$ in the plasma core, i.e., for $u \gg 1$, can be obtained by using $n=\sigma_{*} d u / d x$. In dimensionless quantities $\eta=$

$$
\begin{gathered}
n_{c} \frac{\sigma_{*}\left(x_{w}-x_{1}\right)}{2} \text { and } \gamma=\left(x_{w}-x_{1}\right) \sqrt{\Gamma\left(x=x_{w}\right) \frac{\sigma_{*}}{2 D_{1}}} \text { we get } \\
\eta=\eta_{1}(\gamma) \equiv \gamma \sqrt{1+\frac{\Delta}{\cosh ^{2} \gamma}}
\end{gathered}
$$

where $\Delta=\frac{D_{1}}{D_{0}}-1$.

In the case of a homogeneous plasma diffusivity, $D=$ $D_{0}=D_{1}, \Delta=0$, we have $\eta=\gamma$; i.e., the previous result (see, e.g., [7]), $\Gamma\left(x=x_{w}\right)=D \sigma_{*} n_{c}^{2} / 2$, is recovered. The latter can be understood by taking into account that the density gradient at the plasma edge is $n_{c} / l_{n}$ where $l_{n} \approx$ $2 /\left(n_{c} \sigma_{*}\right)$ is the penetration depth of neutrals. If $\Delta>0$ Eq. (8) predicts that for small $\eta$ and $\gamma$ the inner plasma region with the lower diffusivity $D=D_{0}$ is decisive. Conversely, the layer near the wall with higher $D=D_{1}$ is of the most importance for large $\eta$ and $\gamma$. Figure 2 demonstrates $\eta$ versus $\gamma$ for different $\Delta$. One can see, if $\Delta$ is sufficiently large, the $\eta(\gamma)$ dependence is a nonmonotonic $N$-like one with a negative slope in the transition zone between the regions considered above. The critical value of $\Delta, \Delta_{\mathrm{cr}}$, at which the nonmonotony appears, can be determined from the requirement that both $d \eta / d \gamma$ and $d^{2} \eta / d \gamma^{2}$ reduce to zero at the same $\gamma_{\mathrm{cr}}$. This leads to the equation $1.5=\gamma_{\mathrm{cr}}\left(\tanh \gamma_{\mathrm{cr}}-1 / \sinh 2 \gamma_{\mathrm{cr}}\right)$ providing $\gamma_{\mathrm{cr}} \approx 1.718$ and $\Delta_{\mathrm{cr}}=\cosh ^{2} \gamma_{\mathrm{cr}} /\left(\gamma_{\mathrm{cr}} \tanh \gamma_{\mathrm{cr}}-1\right) \approx 13.54$.

Stability analysis. - In the case of a nonmonotonic $\eta(\gamma)$ dependence three stationary values of the particle loss to the wall are possible for the same plasma density if this belongs to a certain range (see Fig. 2). In order to analyze the consequences of this the stability of stationary states is considered. For this purpose we sum up Eqs. (1) and (2) and integrate the result over the plasma volume by taking into account that (i) the amount of neutrals inside hot plasmas in question is negligibly small compared with

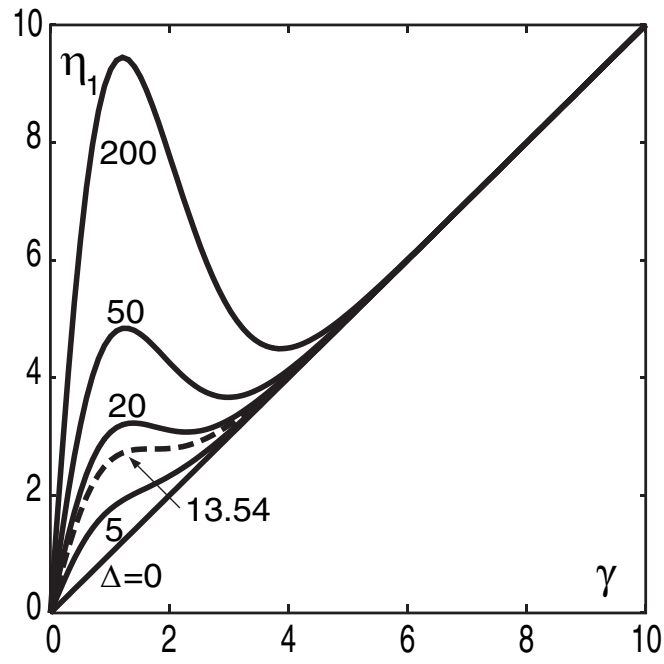

FIG. 2. The plasma density far from the wall versus the plasma flux to the wall for different levels of the discontinuity in the diffusivity of charged particles. 


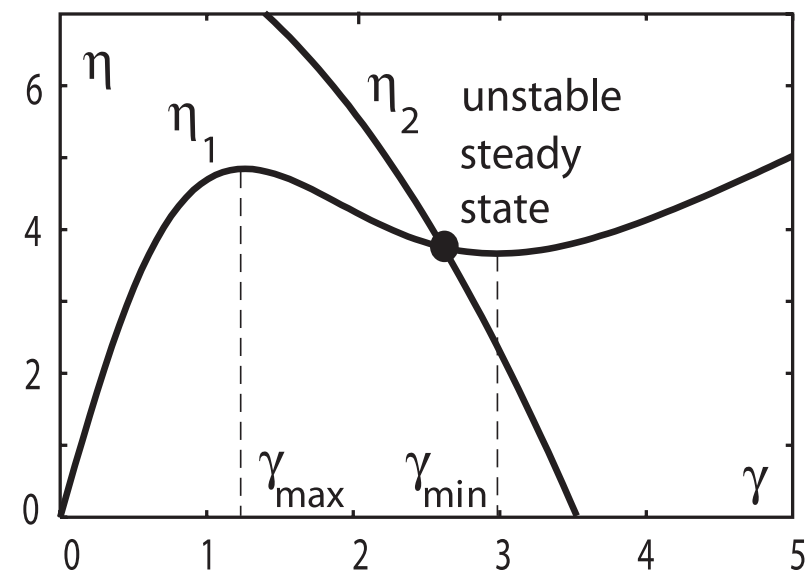

FIG. 3. An unstable stationary state of the plasma-wall system with $\Delta=50, \alpha=1.5$, and $\eta_{0}=8.3$.

that of charged particles and (ii) the particle fluxes disappear far from the wall. With Eq. (3) this leads to the charged particle balance:

$$
x_{w} \frac{d n_{c}}{d t} \approx \mu C_{w}-\Gamma\left(x=x_{w}\right) .
$$

This takes into account that $n$ varies only in the region where neutrals are ionized, which is much thinner then the plasma dimension $x_{w}$, e.g., the minor radius of the plasma column in a tokamak. By using Eq. (4) one gets $d\left(x_{w} n_{c}+\right.$ $\left.C_{w}\right) / d t=0$ or, after the time integration, the conservation law for the total number of particles in the plasma-wall system: $x_{w} n_{c}+C_{w}=$ const $\equiv x_{w} n_{0}$. This can be used by rewriting Eq. (9) in the dimensionless variables $\eta$ and $\gamma$ introduced above:

$$
\frac{d \eta}{d t}=\mu\left(\eta_{0}-\eta-\frac{\gamma^{2}}{\alpha}\right)
$$

with $\eta_{0}=n_{0} \sigma_{*}\left(x_{w}-x_{1}\right) / 2$ and $\alpha=\mu\left(x_{w}-x_{1}\right) x_{w} / D_{1}$.

The conditions for instability one finds by linearizing Eq. (10) for small perturbations of $\eta$ proportional to $\exp (\nu t)$. This provides the growth rate of perturbations:

$$
\nu=-\mu\left(1+\frac{2 \gamma}{\alpha} \frac{\partial \gamma}{\partial \eta}\right)
$$

Only stationary states with $\partial \gamma / \partial \eta<0$ can be unstable, i.e., have $\nu>0$, if the desorption rate is sufficiently slow:

$$
\frac{\partial \gamma}{\partial \eta} \leq-\frac{\alpha}{2 \gamma}
$$

Moreover, under certain conditions this unstable state can be a single stationary one. In order to find when this takes place we get from Eq. (10) for a steady state:

$$
\eta=\eta_{2}(\gamma) \equiv \eta_{0}-\gamma^{2} / \alpha
$$

The stationary parameters are determined by the intersection of the curves $\eta_{1}(\gamma)$ and $\eta_{2}(\gamma)$; see Fig. 3. Thus for the

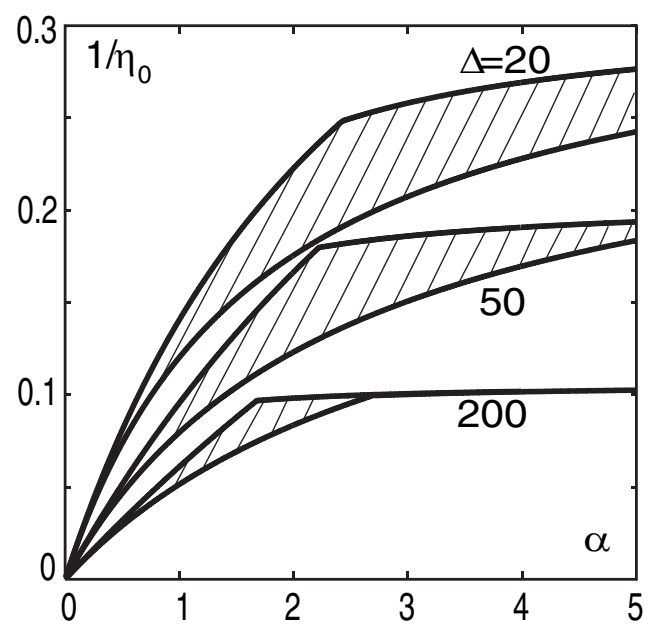

FIG. 4. Regions in the $\left(\alpha, \eta_{0}\right)$ plane where the plasma-wall system in question has a single unstable stationary state.

uniqueness of the unstable stationary state $(\gamma, \eta)$, the conditions

$$
\eta_{1}\left(\gamma_{\max }\right)<\eta_{2}\left(\gamma_{\max }\right), \quad \eta_{1}\left(\gamma_{\min }\right)>\eta_{2}\left(\gamma_{\min }\right)
$$

should be satisfied. Inequalities (12) and (14) define the region in the $\left(\alpha, \eta_{0}\right)$ space where the system in question has a single unstable stationary state. Figure 4 demonstrates these regions for different parameters $\Delta$. They become smaller for very large $\Delta$ because the slope of the curve $\eta_{1}$ is too negative in the instability region and three intersections with the curve $\eta_{2}$ become more probable.

According to the theory of nonlinear systems time evolution of a system with a single unstable stationary state can represent a limit cycle with self-sustained oscillations modeled numerically in the next section.

Numerical modeling. - We consider a plasma sheet enclosed between two parallel walls posed at $x= \pm x_{w}$. A finite $e$-folding length of $1 \mathrm{~cm}$ has been assumed as the boundary condition at the walls. Figure 5 shows the variation of the parameter $\gamma^{2}=\left[\sigma_{*}\left(x_{w}-x_{1}\right)^{2} / 2 D_{1}\right] \Gamma\left(x=x_{w}\right)$ with the dimensionless time $\tau=t D_{0} / x_{w}^{2}$ computed by

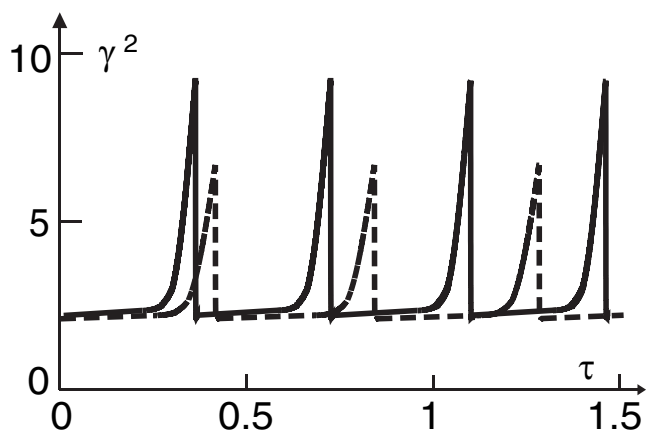

FIG. 5. Time variation of the charged particle loss to the wall computed for the constant (dashed curve) and bell-like (solid curve) temperature profiles. 


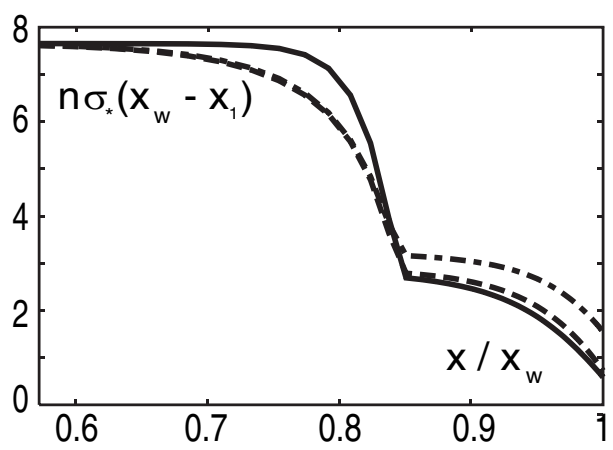

FIG. 6. The plasma density profiles during self-sustained oscillations: at a quasistationary stage with low particle losses from the plasma (solid curve), shortly before (dash-dotted curve) and after (dashed curve) a spike in the plasma loss.

integrating Eqs. (1)-(7) numerically for the same $\Delta, \alpha$, and $\eta_{0}$ used to find the curves in Fig. 3 and $x_{1} / x_{w}=0.85$. The dashed curve was found for the constant temperature $T(r)=200 \mathrm{eV}$; the solid one for a typical for tokamaks bell-like temperature profile $T(r) \sim \exp \left(-x^{2} / x_{T}^{2}\right)$, with $T(0) \approx 1500 \mathrm{eV}$ and $x_{T}=0.6 x_{w}$ [8]. The temperature dependence of the rate coefficients $k_{i}$ and $k_{c x}$ (see, e.g., Ref. [8]) results in their variation through the plasma in the ranges $1.7 \times 10^{-8}-3.3 \times 10^{-8}$ and $4 \times 10^{-8}-9 \times$ $10^{-8} \mathrm{~cm}^{3} / \mathrm{s}$, respectively.

One can see that self-sustained nonlinear oscillations develop in the plasma-wall system under consideration, as well as in the case when a realistic temperature profile and, thus, spatially changing $k_{i}$ and $k_{c x}$ are taken into account. During the long quasistationary phase of low particle losses the particle content in the plasma slowly increases due to desorption of neutrals from the wall. This gradually leads to the increase of the particle outflow from the plasma. This process becomes explosive, however, when neutrals are predominantly ionized in the edge layer with the high transport. Under critical conditions the particle outflow suddenly results in a nearly instantaneous "devastation" of this layer, which cannot be compensated by the slow diffusion of charged particles from the plasma core and neutral desorption from the wall. As a result the plasma density at the edge and the particle loss drop abruptly. The corresponding modifications in the plasma density profile are displayed in Fig. 6.

Our calculations show that self-sustained oscillations are preserved if the diffusivity behavior is relaxed to a continuous transition between very different transport levels or if a cylindrical geometry is assumed.

This study was partly supported by the German Research Society within SFB 591.

*Electronic address: www.fz-juelich.de/ipp

[1] F. Wagner et al., Phys. Rev. Lett. 49, 1408 (1982).

[2] A. Loarte et al., Phys. Rev. Lett. 83, 3657 (1999).

[3] Y. Takeiri et al., Plasma Phys. Controlled Fusion 42, 147 (2000).

[4] P. C. Stangeby, The Plasma Boundary of Magnetic Fusion Devices (Institute of Physics Publishing, Bristol, 2000).

[5] G. Federici et al., Nucl. Fusion 41, 1967 (2001).

[6] B. Lehnert, Nucl. Fusion 8, 173 (1968).

[7] M.Z. Tokar, Plasma Phys. Controlled Fusion 35, 1119 (1993).

[8] J. Wesson, Tokamaks (Claredon Press, Oxford, 2004), 3rd ed.

[9] J. Weiland, Collective Modes in Inhomogeneous Plasma (Institute of Physics Publishing, Bristol, 2000).

[10] M.Z. Tokar, Phys. Rev. Lett. 91, 095001 (2003).

[11] Ph. Ghendrih et al., Plasma Phys. Controlled Fusion 38, 1653 (1996).

[12] I. I. Beilis, M. Keidar, and S. Goldsmith, Phys. Plasmas 4, 3461 (1997). 